\title{
Nonlinear Control of Hydraulic Robots
}

\author{
Mohammad Reza Sirouspour, Septimiu E. Salcudean
}

\begin{abstract}
This paper addresses the control problem of hydraulic robot manipulators. The backstepping design methodology is adopted to develop a novel nonlinear position tracking controller. The tracking errors are shown to be exponentially stable under the proposed control law. The controller is further augmented with adaptation laws to compensate for parametric uncertainties in the system dynamics. Acceleration feedback is avoided by using two new adaptive and robust sliding type observers. The adaptive controllers are proven to be asymptotically stable via Lyapunov analysis. Simulation and experimental results performed with a hydraulic Stewart platform demonstrate the effectiveness of the approach.
\end{abstract}

Keywords - Hydraulic robots, control of robot manipulators, adaptive/nonlinear control, backstepping.

\section{INTRODUCTION}

$\mathrm{H}$ Y draulic robots and machinery are widely used in the construction and mining industries $\Gamma$ as well as in motion simulators. They have rapid responses and high power-to-weight ratios suitable for many applications. High performance controllers can have a significant impact on the effectiveness of hydraulic robots. Furthermore $\mathrm{T}$ the potential complexity of such controllers is becoming less and less of an implementation issue due to the inexpensive and powerful processors available today for real-time control.

In general Tthe control of hydraulic manipulators is more challenging than that of their electrical counterparts. It might seem that a potentially effective way of increasing the performance of hydraulic robots is to consider control methods that neglect actuator dynamics but incorporate the manipulator rigid body dynamics $\Gamma$ such as computed

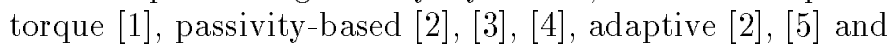
robust $[6] \Gamma[7] \Gamma[8]$ control methods. However $\Gamma$ this is not the case in general. Unlike their electrical counterparts that resemble force sources Thydraulic actuators resemble velocity sources. They also exhibit significant nonlinear characteristics. Therefore $\Gamma$ the above control methods cannot be applied effectively to hydraulic manipulators as hydraulic actuators cannot accurately apply forces or torques over a significant dynamic range.

Actuator dynamic models have been successfully incorporated in the controller design for rigid link electrically driven (RLED) robots to improve position tracking performance. The complete dynamics of the manipulators $\Gamma$ including their actuators $\Gamma$ are third order nonlinear differential equations. [9] used feedback linearization to linearize and decouple these dynamics. [10] developed an adaptive controller for RLED manipulators that does not require acceleration feedback. [11] and [12] and other papers also

The authors are with the Robotics and Control Laboratory, Department of Electrical and Computer Engineering, University of British Columbia, Canada. Emails: shahins@ece.ubc.ca, tims@ece.ubc.ca. considered the adaptive control of RLED robots based on models that include actuator dynamics.

While actuator dynamics are generally linear in RLED robots and can be ignored in many cases due to their fast time constants $\Gamma$ they are highly nonlinear and dominant in hydraulic manipulators. Therefore $\Gamma$ the incorporation of these dynamics in the design of controllers is of critical importance in hydraulic robots. Research in the area of hydraulic systems has mainly focused on the control of single-rod hydraulic actuators $\Gamma$ e.g. see $[13] \Gamma[14] \Gamma[15]$. In particular $\Gamma[13]$ developed a nonlinear position tracking controller for hydraulic servo-systems following the backstepping approach. There are only a few papers that address the control of robot manipulators driven by hydraulic actuators. In [16] Tthe authors established a simplified model in a standard form suitable for the application of singular perturbation methods. No experimental or numerical results are presented in this work. A decentralized adaptive controller was proposed to control a hydraulic manipulator in [17] $\Gamma[18]$. The use of pressure feedback in the control of a Stewart type hydraulic manipulator was proposed in [19]. HoweverT these approaches lack stability proofs that are important from both theoretical and implementation points of view. Only recently $\mathrm{s}$ simultaneous to this work $\Gamma$ [20] proposed a Lyapunov-based adaptive controller for hydraulic robots.

The backstepping design methodology [21]Г[22] has become increasingly popular in the control community. For some recent applications of this method see [23]Г[24]. In this paperTbackstepping is adopted to develop a novel nonlinear controller for hydraulic manipulators. Both rigid body and actuator dynamics are incorporated into the design. The controller is also extended to compensate for parametric uncertainties in the system dynamicsTincluding hydraulic and rigid body dynamics. Two types of observers are developed to avoid the use of acceleration feedback in the proposed adaptive control laws. The first observer is an extension of the passivity-based observers proposed by [3] $\mathrm{C}$ to the case in which the system parameters are unknown. The concept of sliding observers [25] is also adopted to develop a robust acceleration observer. The tracking errors are proven to converge to zero asymptotically using Lyapunov analysis. It can be shown that these errors remain bounded in the presence of Coulomb friction in the actuators. The bounds on the tracking errors are adjustable by the controller gains.

The main differences between this work and the adaptive controller introduced in [20] are the following: (i) the adaptive controller / adaptive observer proposed here uses the same set of estimated rigid body parameters in the observer and controller $\Gamma$ as opposed to the use of two distinct sets of parameter estimates and adaptation laws in [20]; and 
(ii) the introduction of an adaptive control method with a robust observer that is simpler to implement because it has reduced computational complexity. The form of the control laws and the observers are different from those of [20].

PositionTvelocity and hydraulic pressure measurements are required for the implementation of the proposed controllers. Simulation and experimental results for a hydraulic Stewart platform are presented to show the effectiveness of the approach.

The paper is organized as follows. System dynamicsTincluding rigid body and hydraulic dynamics are presented in Section II. In Section III a nonlinear controller is proposed assuming that the dynamics are known exactly. The adaptive control of hydraulic robots is addressed in Section IV for the cases in which the robot dynamics are subject to parametric uncertainty. In Section $\mathrm{V}$ simulation results are presented. The experimental evaluation of the controllers is discussed in Section VI. FinallyTconclusions are drawn in Section VII.

\section{Manipulator/Actuators Dynamics}

The dynamics of an $n$-link robot with rigid links are governed by a second-order nonlinear differential equation

$$
D(q) \ddot{q}+C(q, \dot{q}) \dot{q}+G(q)=\tau
$$

where $q \in R^{n}$ is a vector of generalized joint positions and $\tau \in R^{n}$ is a vector of generalized joint torques. $D(q) \in$ $R^{n \times n}$ is the manipulator mass matrix $\Gamma(q, \dot{q}) \in R^{n \times n}$ contains coriolis and centripetal terms and $G(q) \in R^{n}$ represents gravitational effects. Unlike electrically driven manipulators $\Gamma$ hydraulic robots exhibit significant nonlinear actuator dynamics. Assuming a three-way valve configurationTthese dynamics can be written in the following form $\Gamma$

$$
\dot{\tau}=f(q, \dot{q})+g(q, \tau, u)
$$

where $u$ is the control command vector and $f \Gamma g$ are nonlinear functions of $q \Gamma \dot{q}$ and $\tau$. The detailed expressions for $f$ and $g$ are given in Appendix $A$.

The matrices describing the rigid body dynamics in (1) satisfy the following properties [3]:

$$
\begin{array}{ccc}
\text { (i) } & x^{T}(\dot{D}(q)-2 C(q, \dot{q})) x=0 & \forall x \in R^{n} \\
\text { (ii) } & C(q, x) y=C(q, y) x \quad \forall q, x, y \in R^{n} \\
\text { (iii) } & \exists D_{m}, D_{M} & \text { s.t. } 0<D_{m} \leq\|D(q)\| \leq D_{M}<\infty \\
& & \forall q \in R^{n} \\
\text { (iv) } \exists C_{M} & \text { s.t. } \quad\|C(q, x)\| \leq C_{M}\|x\| \quad \forall q, x \in R^{n} \\
\text { (v) } \exists G_{M} & \text { s.t. }\|G(q)\| \leq G_{M} \quad \forall q \in R^{n}
\end{array}
$$

which are exploited in deriving the proposed control laws. According to (1) and (2) Tthe overall actuator/manipulator dynamics are governed by a set of third-order nonlinear differential equations.

\section{Nonadaptive Controller}

In this section The backstepping design methodology [21] is adopted to derive a nonlinear position tracking controller for hydraulic manipulators in the case in which the system parameters are known.

Theorem 1: Consider the system described by (1) $\Gamma(2)$ with the control law given by the solution $u$ of the following algebraic equation:

$$
g(q, \tau, u)=-f(q, \dot{q})-\Gamma^{-1} s+\dot{\tau}_{d}-K_{\tau} \tilde{\tau}
$$

and

$$
\tau_{d}=D(q) \ddot{q}_{r}+C(q, \dot{q}) \dot{q}_{r}+G(q)-K_{p} e-K_{d} s
$$

with

$$
\begin{aligned}
& e=q-q_{d}, \quad \dot{q}_{r}=\dot{q}_{d}-\Lambda e, \\
& s=\dot{q}-\dot{q}_{r}=\dot{e}+\Lambda e, \quad \tilde{\tau}=\tau-\tau_{d}
\end{aligned}
$$

where $K_{p} \Gamma K_{d} \Gamma K_{\tau} \Gamma \Gamma$ and $\Lambda$ are positive definite diagonal matrices $\Gamma$ and $q_{d} \in R^{n}$ and $\dot{q}_{d} \in R^{n}$ are the desired joint position and velocity trajectories Trespectively.

Then $\underline{0}$ is an exponentially stable equilibrium point for the state $\tilde{x}=\left[\begin{array}{lll}e^{T} & s^{T} & \tilde{\tau}^{T}\end{array}\right]^{T}$ of (1) I(2)I(4)I(5).

Remark: From the expression of $g$ from Appendix A Tit can be seen that (4) can be easily solved for $u$.

Proof: Substituting (5) into (1) yields the following error dynamics $\Gamma$

$$
D(q) \dot{s}+C(q, \dot{q}) s+K_{d} s+K_{p} e=\tilde{\tau}
$$

Note that the effect of actuator dynamics emerges as a non-zero $\tilde{\tau} \Gamma$ as the controller reduces to a passivity-based controller [26] in the absence of actuator dynamics. Let $V_{1}$ be defined as

$$
V_{1}=\frac{1}{2} s^{T} D(q) s+\frac{1}{2} e^{T} K_{p} e
$$

It can be shown that the derivative of $V_{1}$ along trajectories of the closed loop system becomes

$$
\dot{V}_{1}=-s^{T} K_{d} s-e^{T} \Lambda K_{p} e+s^{T} \tilde{\tau}
$$

where (7) and the properties given in (3) have been used. Following the backstepping methodology $\Gamma V_{2} \Gamma$ which is a Lyapunov function for the closed-loop system $\Gamma$ is defined as

$$
V_{2}=V_{1}+\frac{1}{2} \tilde{\tau}^{T} \Gamma \tilde{\tau}
$$

with $\Gamma>0$ diagonal. Note that:

$$
\alpha_{m}\|\tilde{x}\|^{2}<V_{2}<\alpha_{M}\|\tilde{x}\|^{2}, \quad \alpha_{m}, \alpha_{M}>0
$$

By taking the derivative of (10) and using the control law (4) Tone can write

$$
\dot{V}_{2}=-s^{T} K_{d} s-e^{T} \Lambda K_{p} e-\tilde{\tau}^{T} \Gamma K_{\tau} \tilde{\tau}<-\beta\|\tilde{x}\|^{2}
$$


with $\beta>0$. Therefore $T$ the system is exponentially stable in the Lyapunov sense. This means that the position tracking error converges to zero exponentially. FurthermoreT since $s=\dot{e}+\Lambda e \Gamma$ the velocity tracking error is also exponentially stable. Note that in the realization of (4) one needs to compute $\dot{\tau}_{d}$ which is equal to

$$
\begin{aligned}
\dot{\tau}_{d} & =\dot{D}(q) \ddot{q}_{r}+D(q) \dddot{q}_{r}+C(q, \dot{q}) \ddot{q}_{r}+\dot{C}(q, \dot{q}, \ddot{q}) \dot{q}_{r} \\
& +\dot{G}(q)-K_{p} \dot{e}-K_{d} \dot{s}
\end{aligned}
$$

Since $\dddot{q}_{r}=\dddot{q}_{d}-\Lambda \ddot{e} \Gamma \dot{s}=\ddot{e}+\Lambda \dot{e}$ and $\dot{C}$ are functions of $\ddot{q} \Gamma$ link accelerations appear in the proposed control law. However $\Gamma$ if $\tau$ is measured through pressure sensors $\Gamma$ the link accelerations $\ddot{q}$ can be obtained from position Tvelocity and pressure measurements using

$$
\ddot{q}=D(q)^{-1}[\tau-C(q, \dot{q}) \dot{q}-G(q)]
$$

Thus $q \Gamma \dot{q}$ and $\tau$ are required to implement the proposed control law that leads to exponentially stable tracking errors.

Remark: Since the system dynamics are fully known and the states are assumed to be measured $\mathrm{f}$ feedback linearization could also be used to derive a stabilizing controller. [9] adopted this approach to develop a controller for electrically driven manipulators in the presence of linear actuator dynamics.

\section{Adaptive Controller}

The control law derived in the previous section requires full knowledge of the system parameters. However $\Gamma$ the manipulator rigid body dynamics are uncertain and subject to changesTe.g. due to an unknown variable payload. It is also difficult to measure some of the manipulator's parameters. Moreover The hydraulic parameters are usually unknown and time varying. In this section the nonlinear controller proposed in Section III is extended to compensate for parametric uncertainties in the system dynamics. To deal with uncertainties in rigid body dynamicsTthe linear parameterization of manipulator dynamics is used [26]

$$
D(q) \ddot{q}+C(q, \dot{q}) \dot{q}+G(q)=Y(q, \dot{q}, \ddot{q}) \theta
$$

where $Y(q, \dot{q}, \ddot{q})$ is a regressor matrix and $\theta \in R^{m}$ is the vector of unknown parameters. SimilarlyTas shown in $A p$ pendix ATthe hydraulic dynamics (2) can be written as

$$
\dot{\tau}=f_{0}(q, \dot{q}) \gamma_{1}+g_{0}(q, \tau, u) \gamma_{2}
$$

where $\gamma_{1}=\left[\begin{array}{lll}\gamma_{1}^{1} & \ldots & \gamma_{1}^{n}\end{array}\right]^{T} \Gamma \gamma_{2}=\left[\begin{array}{lll}\gamma_{2}^{1} & \ldots & \gamma_{2}^{n}\end{array}\right]^{T}$ are two sets of hydraulic parameters and $f_{0} \Gamma g_{0}$ are defined as

$$
\begin{aligned}
f_{0}(q, \dot{q}) & =\operatorname{diag}\left\{f_{0}^{i}\left(q^{i}, \dot{q}^{i}\right)\right\} \\
g_{0}(q, \tau, u) & =\operatorname{diag}\left\{g_{0}^{i}\left(q^{i}, \tau^{i}, u^{i}\right)\right\}
\end{aligned}
$$

In the non-adaptive controller $\Gamma$ (14) was used to compute joint accelerations from joint positions and velocities and hydraulic pressure measurements. This can not be done if $D(q) \Gamma C(q, \dot{q})$ and $G(q)$ are not known. To deal with this problem novel adaptive and robust observers are introduced. The following Lemma [27] will be used in the stability proofs.

Lemma 1: Consider the scalar function $\alpha=(\theta-\hat{\theta})^{T}(\rho-\hat{\theta}) \Gamma$ with $\theta, \hat{\theta}, \rho \in R^{n}$ and $a^{i} \leq \theta^{i} \leq b^{i}$.

If $\dot{\hat{\theta}}=\kappa(a, b, \rho) \rho$ T where $\kappa(a, b, \rho)$ is a diagonal matrix with entries

$$
\kappa^{i}(a, b, \rho)= \begin{cases}0 & \text { if } \hat{\theta}^{i} \leq a^{i} \Gamma \rho^{i} \leq 0 \\ 0 & \text { if } \hat{\theta}^{i} \geq b^{i} \Gamma \rho^{i} \geq 0 \\ 1 & \text { otherwise }\end{cases}
$$

then $\alpha \leq 0$

\section{A. Adaptive Controller/Adaptive Observer}

The first solution is an adaptive controller using an adaptive passivity-based observer. Before stating the result Tthe following notation must be defined:

$$
\begin{aligned}
& \dot{q}_{r}=\dot{q}_{d}-\Lambda_{1}\left(\hat{q}-q_{d}\right)=\dot{q}_{d}-\Lambda_{1}(e-\tilde{q}) \\
& \dot{q}_{o}=\dot{\hat{q}}-\Lambda_{2}(q-\hat{q})=\dot{\hat{q}}-\Lambda_{2} \tilde{q} \\
& s_{1}=\dot{q}-\dot{q}_{r}=\dot{e}+\Lambda_{1}(e-\tilde{q}) \\
& s_{2}=\dot{q}-\dot{q}_{o}=\dot{\tilde{q}}+\Lambda_{2} \tilde{q}
\end{aligned}
$$

where $\hat{q} \in R^{n}$ is the estimated value of $q \Gamma e=q-q_{d} \Gamma$ and $\tilde{q}=q-\hat{q}$ are position tracking and observation errors $\Gamma$ respectively. $\Lambda_{1}, \Lambda_{2}>0$ are diagonal. Note that in the definitions of $\dot{q}_{r}$ and $\dot{q}_{o} \Gamma \dot{q}$ has been replaced by $\dot{q}_{d}$ and $\dot{\hat{q}}$. This will be shown to eliminate the need for acceleration feedback.

Theorem 2: Consider the system described by (1) $\Gamma(2) \Gamma$ the observer dynamics

$$
\begin{aligned}
& \dot{\hat{q}}=z+\Lambda_{2} \tilde{q} \\
& \dot{z}=\hat{D}(q)^{-1}\left[\tau-\hat{C}(q, \dot{q}) \dot{q}_{o}-\hat{G}(q)+L_{p} \tilde{q}+K_{d} s_{1}\right. \\
& \left.\quad+K_{d}^{\prime} s_{2}\right]
\end{aligned}
$$

and the controller obtained by solving the following algebraic equation

$$
g_{0}(q, \tau, u) \hat{\gamma}_{2}=\dot{\tau}_{d}-f_{0}(q, \dot{q}) \hat{\gamma}_{1}-\Gamma_{\tau}^{-1} s_{1}-K_{\tau} \tilde{\tau}
$$

where

$$
\begin{aligned}
\tau_{d} & =\hat{D}(q) \ddot{q}_{r}+\hat{C}\left(q, \dot{q}_{r}\right) \dot{q}_{r}+\hat{G}(q)-K_{d}\left(s_{1}-s_{2}\right)-K_{p} e \\
& =Y_{1}\left(q, \dot{q}_{r}, \ddot{q}_{r}\right) \hat{\theta}-K_{d}\left(s_{1}-s_{2}\right)-K_{p} e
\end{aligned}
$$

with unknown rigid body parameter adaptation law

$$
\dot{\hat{\theta}}=-\kappa_{\theta} \Gamma^{-1}\left[Y_{1}^{T}\left(q, \dot{q}_{r}, \ddot{q}_{r}\right) s_{1}+Y_{2}^{T}\left(q, \dot{q}, \dot{q}_{o}, \ddot{q}_{o}\right) s_{2}\right]
$$

where

$$
\begin{aligned}
& Y_{1}\left(q, \dot{q}_{r}, \ddot{q}_{r}\right) \hat{\theta}=\hat{D}(q) \ddot{q}_{r}+\hat{C}\left(q, \dot{q}_{r}\right) \dot{q}_{r}+\hat{G}(q) \\
& Y_{2}\left(q, \dot{q}, \dot{q}_{o}, \ddot{q}_{o}\right) \hat{\theta}=\hat{D}(q) \ddot{q}_{o}+\hat{C}(q, \dot{q}) \dot{q}_{o}+\hat{G}(q)
\end{aligned}
$$


and with hydraulic parameter adaptation laws

$$
\begin{aligned}
& \dot{\hat{\gamma}}_{1}=\kappa_{\gamma_{1}} \Gamma_{\gamma_{1}}^{-1} \Gamma_{\tau} f_{0}(q, \dot{q}) \tilde{\tau} \\
& \dot{\hat{\gamma}}_{2}=\kappa_{\gamma_{2}} \Gamma_{\gamma_{2}}^{-1} \Gamma_{\tau} \Pi\left(\frac{\tilde{\tau}}{\hat{\gamma}_{2}}\right)\left(\dot{\tau}_{d}-f_{0}(q, \dot{q}) \hat{\gamma}_{1}-\Gamma_{\tau}{ }^{-1} s_{1}-K_{\tau} \tilde{\tau}\right)
\end{aligned}
$$

where $\Pi\left(\frac{\tilde{\tau}}{\hat{\gamma}_{2}}\right)=\operatorname{diag}\left\{\frac{\tilde{\tau}^{i}}{{\hat{\gamma_{2}}}^{2}}\right\}$. Then $\Gamma$ if the conditions given below in (26) are satisfied $\Gamma \underline{0}$ is an asymptotically stable equilibrium point of the state $\tilde{x}=$ $\left[\begin{array}{lllll}e^{T} & \tilde{q}^{T} & s_{1}^{T} & s_{2}^{T} & \tilde{\tau}^{T}\end{array}\right]^{T}$.

(a) $\underline{\sigma}\left(K_{p}\right) \underline{\sigma}\left(L_{p}\right) \underline{\sigma}\left(\Lambda_{1}\right) \underline{\sigma}\left(\Lambda_{2}\right)>\frac{1}{4} \bar{\sigma}^{2}\left(K_{p}\right) \bar{\sigma}^{2}\left(\Lambda_{1}\right)$

(b) $\|\tilde{x}(0)\| \leq \sqrt{\frac{\alpha_{m}}{3 \alpha_{M}}\left(\frac{\sigma\left(K_{d}\right)-C_{M} \dot{q}_{d m}}{C_{M} \bar{\sigma}\left(\Lambda_{1}\right)}\right)^{2}-\frac{V_{p_{M}}-V_{p_{m}}}{\alpha_{M}}}$

where

$$
\begin{aligned}
& \alpha_{m}=\frac{1}{2} \min \left\{D_{m}, \underline{\sigma}\left(K_{p}\right), \underline{\sigma}\left(L_{p}\right), \underline{\sigma}\left(\Gamma_{\tau}\right)\right\} \\
& \alpha_{M}=\frac{1}{2} \max \left\{D_{M}, \bar{\sigma}\left(K_{p}\right), \bar{\sigma}\left(L_{p}\right), \bar{\sigma}\left(\Gamma_{\tau}\right)\right\} \\
& V_{p_{m}} \leq \frac{1}{2}\left(\tilde{\theta}^{T} \Gamma \tilde{\theta}+\tilde{\gamma}_{1}^{T} \Gamma_{\gamma_{1}} \tilde{\gamma}_{1}+\tilde{\gamma}_{2}^{T} \Gamma_{\gamma_{2}} \tilde{\gamma}_{2}\right) \leq V_{p_{M}}
\end{aligned}
$$

Here $\Gamma \bar{\sigma}($.$) and \underline{\sigma}($.$) denote the maximum and minimum$

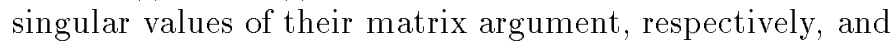
$\dot{q}_{d m}$ is an upper bound on the norm of the desired velocity. The projection gains $\kappa_{\theta} \Gamma \kappa_{\gamma_{1}}$ and $\kappa_{\gamma_{2}}$ are defined as in (18). All of the gains used in the controller and observer $\Gamma$ i.e. $K_{p}, K_{d}, K_{d}^{\prime}, L_{p}, \Gamma, \Gamma_{\gamma_{1}}, \Gamma_{\gamma_{2}}, \Gamma_{\tau}, K_{\tau}$ are constant positive definite diagonal matrices.

Remark 1: The inequality given in (26.b) specifies the boundary of the attraction region that can be enlarged by adjusting the controller gains $\Gamma$ i.e. $K_{d}$ and $K_{p}$. Therefore The closed loop system is semiglobally asymptotically stable. While the controller is guaranteed to be stable $\Gamma$ in practice the parameters should be tuned to achieve the desired performance.

Remark 2: In the above formulation $\Gamma \hat{D} \Gamma \hat{C}$ and $\hat{G}$ are the estimated dynamical matrices corresponding to $\hat{\theta}$. Note that the controller and observer use the same set of estimated parameters which compares favorably to the approach proposed in [20] $\mathrm{T}$ in which different parameter estimates are employed in the controller and observer.

Remark 3: The use of projection gains $\kappa \Gamma$ in the adaptation laws guarantees that the estimate of each parameter remains in a predefined interval $[a, b]$. In particular $\Gamma$ if ${\hat{\gamma_{2}}}^{i}$ becomes zero the control law $u$ in (21) is undefined. This can be avoided by using $a^{i}>0$ for the estimation of $\gamma_{2}^{i}$. Furthermore $\Gamma$ the parameter estimates can not drift because of the upper and lower bounds on their values. Therefore $\Gamma$ parameter adaptation is robust against unmodeled disturbances [27].
Proof: By substituting (22) into (1) the following error dynamics are obtained

$$
\begin{aligned}
& D(q) \dot{s}_{1}+C(q, \dot{q}) s_{1}+K_{d} s_{1}+K_{p} e= \\
& \quad K_{d} s_{2}-C\left(q, s_{1}\right)\left(\dot{q}-s_{1}\right)-Y_{1}\left(q, \dot{q}_{r}, \ddot{q}_{r}\right) \tilde{\theta}+\tilde{\tau}
\end{aligned}
$$

The observer closed-loop dynamics can also be written as

$$
\begin{aligned}
D(q) \dot{s}_{2}+C(q, \dot{q}) s_{2}+K_{d}^{\prime} s_{2}+L_{p} \tilde{q} & = \\
& -K_{d} s_{1}-Y_{2}\left(q, \dot{q}, \dot{q}_{o}, \ddot{q}_{o}\right) \tilde{\theta}
\end{aligned}
$$

where (1) and (20) have been used in deriving (29).

Now Tlet the Lyapunov-like function $V_{1}$ be defined as:

$$
\begin{aligned}
V_{1}= & \frac{1}{2} s_{1}^{T} D(q) s_{1}+\frac{1}{2} e^{T} K_{p} e+\frac{1}{2} s_{2}^{T} D(q) s_{2} \\
& +\frac{1}{2} \tilde{q}^{T} L_{p} \tilde{q}+\frac{1}{2} \tilde{\theta}^{T} \Gamma \tilde{\theta}
\end{aligned}
$$

It can be shown that the derivative of $V_{1}$ along the trajectory of the closed loop system is then given by

$$
\begin{aligned}
\dot{V}_{1} & =-s_{1}{ }^{T} K_{d} s_{1}-s_{2}{ }^{T} K_{d}^{\prime} s_{2}-e^{T} K_{p} \Lambda_{1} e-\tilde{q}^{T} L_{p} \Lambda_{2} \tilde{q} \\
& +e^{T} K_{p} \Lambda_{1} \tilde{q}+s_{1}{ }^{T} \tilde{\tau}+\tilde{\theta}^{T}\left[-\Gamma \dot{\hat{\theta}}-Y_{1}^{T}\left(q, \dot{q}_{r}, \ddot{q}_{r}\right) s_{1}\right. \\
& \left.-Y_{2}^{T}\left(q, \dot{q}, \dot{q}_{o}, \ddot{q}_{o}\right) s_{2}\right]-s_{1}^{T} C\left(q, s_{1}\right)\left(\dot{q}-s_{1}\right)
\end{aligned}
$$

With the adaptation law given in (23) and using Lemma 1 Twe have that

$$
\begin{aligned}
\dot{V}_{1} & \leq-s_{1}^{T} K_{d} s_{1}-s_{2}{ }^{T} K_{d}^{\prime} s_{2}-e^{T} K_{p} \Lambda_{1} e-\tilde{q}^{T} L_{p} \Lambda_{2} \tilde{q} \\
& +e^{T} K_{p} \Lambda_{1} \tilde{q}+s_{1}^{T} \tilde{\tau}-s_{1}^{T} C\left(q, s_{1}\right)\left(\dot{q}_{d}-\Lambda_{1} e+\Lambda_{1} \tilde{q}\right) \\
& \leq-\left(\underline{\sigma}\left(K_{d}\right)-C_{M}\left(\dot{q}_{d m}+\bar{\sigma}\left(\Lambda_{1}\right)\|e\|+\bar{\sigma}\left(\Lambda_{1}\right)\|\tilde{q}\|\right)\right)\left\|s_{1}\right\|^{2} \\
& -\underline{\sigma}\left(K_{d}^{\prime}\right)\left\|s_{2}\right\|^{2}-\underline{\sigma}\left(K_{p}\right) \underline{\sigma}\left(\Lambda_{1}\right)\|e\|^{2}-\underline{\sigma}\left(L_{p}\right) \underline{\sigma}\left(\Lambda_{2}\right)\|\tilde{q}\|^{2} \\
& +\bar{\sigma}\left(K_{p}\right) \bar{\sigma}\left(\Lambda_{1}\right)\|e\|\|\tilde{q}\|+s_{1}^{T} \tilde{\tau} \\
& =H\left(\|e\|,\|\tilde{q}\|,\left\|s_{1}\right\|,\left\|s_{2}\right\|\right)+s_{1}^{T} \tilde{\tau}
\end{aligned}
$$

Note that since

$$
\begin{gathered}
-\underline{\sigma}\left(K_{p}\right) \underline{\sigma}\left(\Lambda_{1}\right)\|e\|^{2}-\underline{\sigma}\left(L_{p}\right) \underline{\sigma}\left(\Lambda_{2}\right)\|\tilde{q}\|^{2}+\bar{\sigma}\left(K_{p}\right) \bar{\sigma}\left(\Lambda_{1}\right)\|e\|\|\tilde{q}\| \\
=-\left[\begin{array}{lll}
\|e\| & \|\tilde{q}\|
\end{array}\right]\left[\begin{array}{cc}
\frac{\sigma}{(}\left(K_{p}\right) \underline{\sigma}\left(\Lambda_{1}\right) & -\frac{1}{2} \bar{\sigma}\left(K_{p}\right) \bar{\sigma}\left(\Lambda_{1}\right) \\
-\frac{1}{2} \bar{\sigma}\left(K_{p}\right) \bar{\sigma}\left(\Lambda_{1}\right) & \underline{\sigma}\left(L_{p}\right) \underline{\sigma}\left(\Lambda_{2}\right)
\end{array}\right] \\
{\left[\begin{array}{c}
\|e\| \\
\|\tilde{q}\|
\end{array}\right]}
\end{gathered}
$$

the condition given in (26.a) and the inequality

$$
\|e\|+\|\tilde{q}\|<\frac{\sigma\left(K_{d}\right)-C_{M} \dot{q}_{d m}}{C_{M} \bar{\sigma}\left(\Lambda_{1}\right)}
$$

guarantee that

$H\left(\|e\|,\|\tilde{q}\|,\left\|s_{1}\right\|,\left\|s_{2}\right\|\right) \leq-\alpha\left(\|e\|^{2}+\|\tilde{q}\|^{2}+\left\|s_{1}\right\|^{2}+\left\|s_{2}\right\|^{2}\right)$

with $\alpha>0$. It is not difficult to show that if (26.b) holds then (34) is also satisfied. 
Following the backstepping approach $\Gamma V_{2} \Gamma$ which is a Lyapunov function for the system dynamicsTis defined as

$$
V_{2}=V_{1}+\frac{1}{2} \tilde{\tau}^{T} \Gamma_{\tau} \tilde{\tau}+\frac{1}{2} \tilde{\gamma}_{1}^{T} \Gamma_{\gamma_{1}} \tilde{\gamma}_{1}+\frac{1}{2} \tilde{\gamma}_{2}^{T} \Gamma_{\gamma_{2}} \tilde{\gamma}_{2}
$$

where $\tilde{\gamma}_{1}=\left[\begin{array}{lll}\tilde{\gamma}_{1}^{1} & \ldots & \tilde{\gamma}_{1}^{n}\end{array}\right]^{T}$ and $\tilde{\gamma}_{2}=\left[\begin{array}{lll}\tilde{\gamma}_{2}^{1} & \ldots & \tilde{\gamma}_{2}^{n}\end{array}\right]^{T}$ are the vectors of hydraulic parameter errors. By taking the derivative of (36) and employing the control law given in (21) Tone can show after some manipulation that

$$
\begin{aligned}
\dot{V}_{2} & \leq H\left(\|e\|,\|\tilde{q}\|,\left\|s_{1}\right\|,\left\|s_{2}\right\|\right)-\tilde{\tau}^{T} \Gamma_{\tau} K_{\tau} \tilde{\tau} \\
& +\tilde{\gamma}_{1}^{T}\left[f_{0}(q, \dot{q}) \Gamma_{\tau} \tilde{\tau}-\Gamma_{\gamma_{1}} \dot{\hat{\gamma}}_{1}\right]+\tilde{\gamma}_{2}^{T}\left[\Gamma_{\tau} \Pi\left(\frac{\tilde{\tau}}{\hat{\gamma}_{2}}\right)\right. \\
& \left.\left(\dot{\tau}_{d}-f_{0}(q, \dot{q}) \hat{\gamma}_{1}-\Gamma_{\tau}^{-1} s_{1}-K_{\tau} \tilde{\tau}\right)-\Gamma_{\gamma_{2}} \dot{\hat{\gamma}}_{2}\right]
\end{aligned}
$$

Using the adaptation laws given in (25) and Lemma 1 the derivative of $V_{2}$ becomes

$$
\begin{aligned}
\dot{V}_{2} & \leq H\left(\|e\|,\|\tilde{q}\|,\left\|s_{1}\right\|,\left\|s_{2}\right\|\right)-\tilde{\tau}^{T} \Gamma_{\tau} K_{\tau} \tilde{\tau} \\
& \leq-\gamma\left(\|e\|^{2}+\|\tilde{q}\|^{2}+\left\|s_{1}\right\|^{2}+\left\|s_{2}\right\|^{2}+\|\tilde{\tau}\|^{2}\right)
\end{aligned}
$$

Thus the position and velocity tracking errors converge to zero asymptotically.

Remark 1: For parameter convergence the condition of persistency of excitation must be satisfied [26].

Remark 2: Inspection of (22) reveals that $\tau_{d}$ does not contain $\dot{q}$. This means that acceleration term $\ddot{q}$ does not appear in $\dot{\tau}_{d}$ and hence in the control law. This is achieved by the particular definition of $\dot{q}_{r}$ in (19) and also by using $s_{1}-s_{2}=\dot{q}_{0}-\dot{q}_{r}$ instead of $s_{1}$ in (22). In summary the proposed controller requires $q \Gamma \dot{q}$ and $\tau$ to be measured.

Remark 3: In order to implement the observer proposed in (20) $\Gamma \hat{D}^{-1}(q)$ must exist. This can be guaranteed by choosing bounds on the estimates of the rigid body parameters.

\section{B. Adaptive Controller/ Robust Observer}

In this subsectionTan adaptive/nonlinear controller utilizing a sliding type observer [25] is proposed that yields globally asymptotically stable tracking errors.

Before stating the result the following variables should be defined:

$$
\dot{q}_{r}=\dot{q}_{d}-\Lambda e, \quad s=\dot{q}-\dot{q}_{r}=\dot{e}+\Lambda e
$$

and $\Lambda>0$ is diagonal.

Theorem 3: Consider the system described by (1) I(2) and the following observer:

$$
\dot{z}=\Gamma_{o} \dot{\tilde{q}}+\Lambda_{o} \operatorname{sgn}(\dot{\tilde{q}})-W^{T}\left(q, \dot{q}_{r}, \hat{\theta}\right) s+\ddot{\bar{q}}
$$

with

$$
\begin{gathered}
W\left(q, \dot{q}_{r}, \hat{\theta}\right)=-\hat{D}(q) \Lambda+\hat{C}\left(q, \dot{q}_{r}\right)-K_{d} \\
\ddot{\bar{q}}=\bar{D}^{-1}[\tau-\bar{C} \dot{q}-\bar{G}]
\end{gathered}
$$

where $z=\dot{\hat{q}}$ is the observed velocity. $\bar{D} \Gamma \bar{C}$ and $\bar{G}$ are constant matrices (rough estimates of dynamical matrices). Let the control law be given by the solution $u$ of the following algebraic equation

$$
g_{0}(q, \tau, u) \hat{\gamma}_{2}=\dot{\tau}_{d}-f_{0}(q, \dot{q}) \hat{\gamma}_{1}-\Gamma_{\tau}^{-1} s-K_{\tau} \tilde{\tau}
$$

where

$\tau_{d}=\hat{D}(q)\left(\ddot{q}_{r}+\Lambda \dot{\tilde{q}}\right)+\hat{C}(q, \dot{\hat{q}}) \dot{q}_{r}+\hat{G}(q)-K_{d}(s-\dot{\tilde{q}})-K_{p} e$

and let the adaptation laws be given by

$$
\dot{\hat{\theta}}=-\kappa_{\theta} \Gamma_{\theta}^{-1} Y^{T}\left(q, \dot{q}, \dot{q}_{r}, \ddot{q}_{r}\right) s
$$

and

$$
\begin{aligned}
& \dot{\gamma}_{1}=\kappa_{\gamma_{1}} \Gamma_{1}^{-1} \Gamma_{\tau} f_{0}(q, \dot{q}) \tilde{\tau} \\
& \dot{\hat{\gamma}}_{2}=\kappa_{\gamma_{2}} \Gamma_{2}^{-1} \Gamma_{\tau} \Pi\left(\frac{\tilde{\tau}}{\hat{\gamma}_{2}}\right)\left(\dot{\tau}_{d}-f_{0}(q, \dot{q}) \hat{\gamma}_{1}-\Gamma_{\tau}{ }^{-1} s-K_{\tau} \tilde{\tau}\right)
\end{aligned}
$$

for the rigid body and hydraulic parameters $\Gamma$ respectively. Then $\underline{0}$ is an asymptotically stable equilibrium point of the state $\tilde{x}=\left[\begin{array}{llll}e^{T} & s^{T} & \dot{\tilde{q}}^{T} & \tilde{\tau}^{T}\end{array}\right]^{T}$. In the above equations $K_{p}, K_{d}, \Lambda_{o}, \Gamma_{\theta}, \Gamma_{1}, \Gamma_{2}, \Gamma_{\tau}, K_{\tau}$ are positive definite diagonal matrices.

Remark: $\tau_{d}$ does not contain any velocity terms. This can be seen from:

$$
\begin{aligned}
& \ddot{q}_{r}+\Lambda \dot{\tilde{q}}=\ddot{q}_{d}-\Lambda\left(\dot{q}-\dot{q}_{d}\right)+\Lambda(\dot{q}-\dot{\hat{q}})=\ddot{q}_{d}-\Lambda\left(\dot{\hat{q}}-\dot{q}_{d}\right) \\
& s-\dot{\tilde{q}}=\dot{q}-\dot{q}_{r}-\dot{q}+\dot{\hat{q}}=\dot{\hat{q}}-\dot{q}_{r}
\end{aligned}
$$

Proof: By substituting (44) into (1) the following closed loop dynamics are obtained

$$
\begin{aligned}
D(q) \dot{s}+C(q, \dot{q}) s & +K_{d} s+K_{p} e= \\
& -Y\left(q, \dot{q}, \dot{q}_{r}, \ddot{q}_{r}\right) \tilde{\theta}-W\left(q, \dot{q}_{r}, \hat{\theta}\right) \dot{\tilde{q}}+\tilde{\tau}
\end{aligned}
$$

Define the Lyapunov-like function $V_{1}$ to be

$$
V_{1}=\frac{1}{2} e^{T} K_{p} e+\frac{1}{2} s^{T} D(q) s+\frac{1}{2} \dot{\tilde{q}}^{T} \dot{\tilde{q}}+\frac{1}{2} \tilde{\theta}^{T} \Gamma_{\theta} \tilde{\theta}
$$

The derivative of $V_{1}$ becomes

$$
\begin{aligned}
\dot{V}_{1} & =s^{T}\left[-Y\left(q, \dot{q}, \dot{q}_{r}, \ddot{q}_{r}\right) \tilde{\theta}-W\left(q, \dot{q}_{r}, \hat{\theta}\right) \dot{\tilde{q}}-C(q, \dot{q}) s\right. \\
& \left.-K_{d} s-K_{p} e+\tilde{\tau}\right]+\frac{1}{2} s^{T} \dot{D}(q) s+e^{T} K_{p}(s-\Lambda e) \\
& +\dot{\tilde{q}}^{T}\left[\ddot{q}-\Gamma_{o} \dot{\tilde{q}}-\Lambda_{o} \operatorname{sgn}(\dot{\tilde{q}})+W^{T}\left(q, \dot{q}_{r}, \hat{\theta}\right) s-\ddot{\bar{q}}\right]+\tilde{\theta}^{T} \Gamma_{\theta} \dot{\tilde{\theta}}
\end{aligned}
$$

which can be written in the following form:

$$
\begin{aligned}
\dot{V}_{1} & =-s^{T} K_{d} s-e^{T} K_{p} \Lambda e-\dot{\tilde{q}}^{T} \Gamma_{o} \dot{\tilde{q}}-\dot{\tilde{q}}^{T}[\ddot{\bar{q}}-\ddot{q} \\
& \left.+\Lambda_{o} \operatorname{sgn}(\dot{\tilde{q}})\right]+\tilde{\theta}^{T}\left[\Gamma_{\theta} \dot{\tilde{\theta}}-Y^{T}\left(q, \dot{q}, \dot{q}_{r}, \ddot{q}_{r}\right) s\right]+s^{T} \tilde{\tau}
\end{aligned}
$$




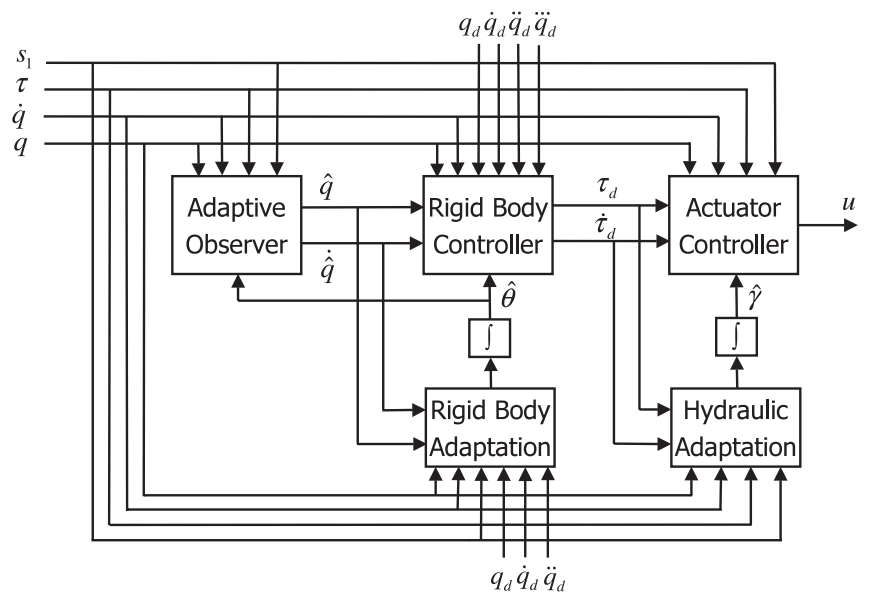

(a)

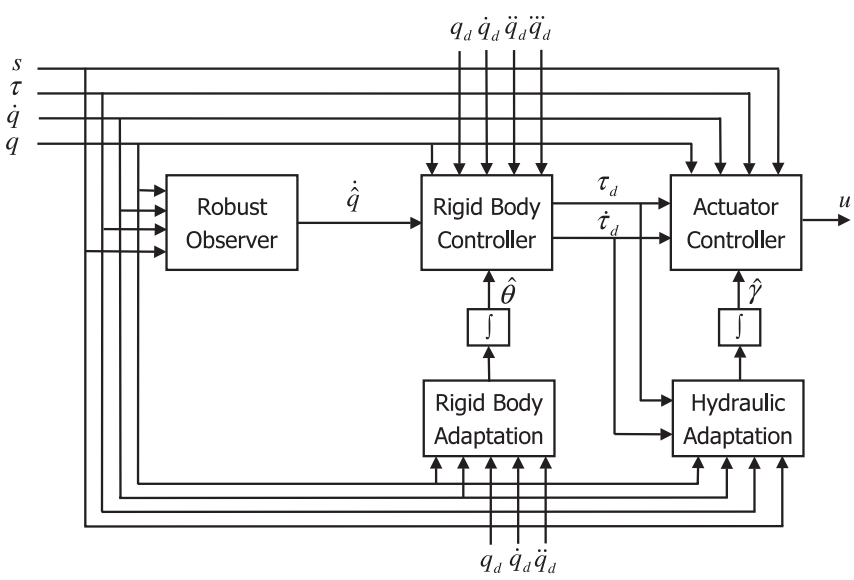

(b)

Fig. 1. Controller implementation block diagrams. (a) Controller with adaptive observer. (b) Controller with robust observer.

With the adaptation law given in $(45) \Gamma \dot{V}_{1}$ becomes

$$
\dot{V}_{1}=-s^{T} K_{d} s-e^{T} K_{p} \Lambda e-\dot{\tilde{q}}^{T} \Gamma_{o} \dot{\tilde{q}}+\Sigma+s^{T} \tilde{\tau}
$$

where $\Sigma=-\dot{\tilde{q}}\left[\ddot{\bar{q}}-\ddot{q}+\Lambda_{o} \operatorname{sgn}(\dot{\tilde{q}})\right]$. It is not difficult to show that

$$
\|\ddot{\bar{q}}-\ddot{q}\| \leq \sigma_{0}+\sigma_{1}\|\dot{q}\|^{2}+\sigma_{2}\|\tau\|+\sigma_{3}\|\dot{q}\| .
$$

with $\sigma_{i}>0$. The properties given in (3) have been exploited in deriving (53). The following choice of $\Lambda_{\circ}$ makes $\Sigma<0$ :

$$
\begin{gathered}
\Lambda_{o}=\operatorname{diag}\left\{\Lambda_{o}^{i}\right\} \\
\Lambda_{o}^{i}=\lambda_{0}^{i}+\lambda_{1}^{i}\|\dot{q}\|^{2}+\lambda_{2}^{i}\|\tau\|+\lambda_{3}^{i}\|\dot{q}\|
\end{gathered}
$$

and $\lambda_{k}^{i}>\sigma_{k}$ for $i=1, \cdots, n$ and $k=0, \cdots, 3$. The rest of the proof is the same as before and will not be presented here.

Note that there are no limitations on the norms of the initial state tracking errors in this approach. HoweverTchattering phenomena which are inherent in sliding mode systems $\Gamma$ can affect stability. For exampleГif high frequency dynamics (e.g. valve dynamics) are excited $\Gamma$ instability could result. The problem could be solved by using a piecewise linear approximation to $\operatorname{sgn}($.$) .$

\section{The Effect of Friction}

In the controllers proposed in this paperTfriction in the hydraulic actuators has been neglected. It is easy to handle viscous friction since it acts as additional damping in the system. It can also be shown that in the presence of Coulomb friction $\Gamma$ the tracking errors do not converge to zero but remain bounded. The error bounds can be reduced by increasing the gains. The proof will be omitted here.

\section{Simulation Results}

Simulations have been performed to investigate the effectiveness of the proposed controllers and to obtain guidelines for experimentation. For this purpose $\mathrm{Ta}$ realistic model of the experimental setup $\Gamma$ a hydraulic Stewart-type platform $\Gamma$ has been used (see Appendices $A$ and $B$ ). The system parameters were selected based upon their actual values and are given in Table I.

In the simulations and experiments conducted for this paperTa task-space control strategy has been followed. The advantage of this approach is that the dynamical matrices have simpler forms in these coordinates for parallel manipulators such as the Stewart platform. HoweverTthe forward kinematics problem must be solved on-line to convert the measured link positions to robot positions in task-space coordinates. Newton's method was utilized for this purpose. The control algorithms and the robot dynamics were all implemented using the Matlab Simulink ${ }^{T M}$ toolbox. The implementation block diagrams of the controllers are shown in Figures 1(a) and 1(b).

Extensive simulations showed similar performance for both of the proposed adaptive control methods Tthus only the results obtained for the controller using the robust observer are presented here. The system parameters were initially set to values different from those used in the model to investigate the ability of the controllers to cope with parametric uncertainties. The reference trajectory was chosen to be $x_{d}=0.02 \sin (2 \pi t)+0.01 \sin (4 \pi t)+0.01 \sin (6 \pi t) \Gamma y_{d}=$ $0 \Gamma z_{d}=0.02 \sin (2 \pi t)+0.01 \sin (4 \pi t) \Gamma \psi_{d}=0.0873 \sin (2 \pi t)+$ $0.0349 \sin (4 \pi t) \Gamma \theta_{d}=0.0524 \sin (2 \pi t)+0.0175 \sin (4 \pi t) \Gamma$ $\phi_{d}=0.0524 \sin (2 \pi t)+0.0175 \sin (4 \pi t)$. Positions and angles are expressed in meters and radiansTrespectively. The tracking errors clearly converge to zero in all coordinates as shown in Figure 2. The profiles of the parameter estimates are given in Figure 3 . The parameter adaptation laws were activated after $t=0.5 \mathrm{~s}$. Both rigid body and hydraulic parameters converge to their actual valuesTeven though the parameter convergence is not guaranteed in theory. The estimates of $I_{x}, I_{y}$ reach their boundaries during some periods of the simulation as seen in Figure 3.

In summary The controller with the robust observer com- 


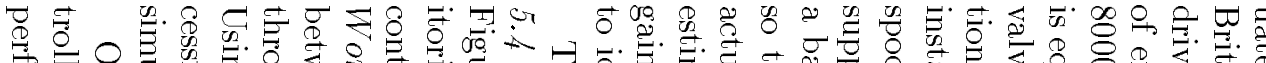

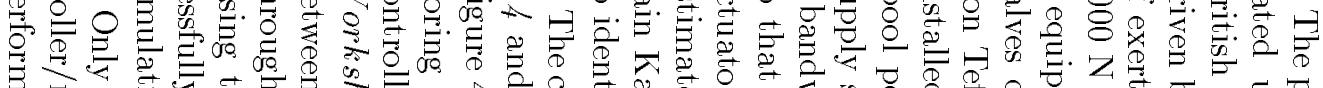

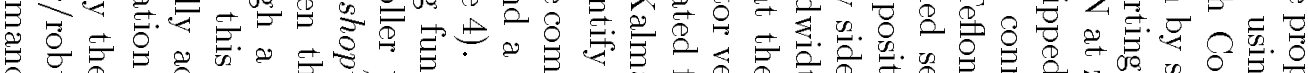

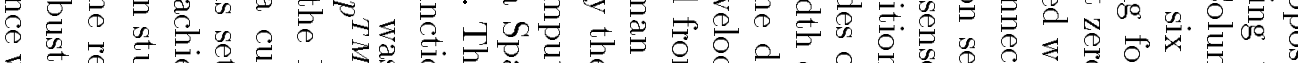

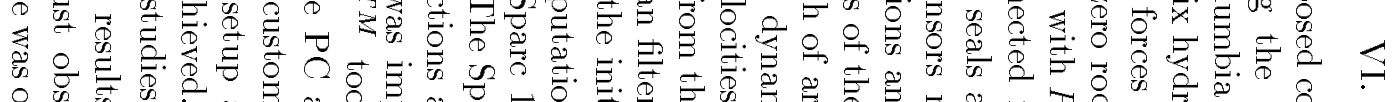

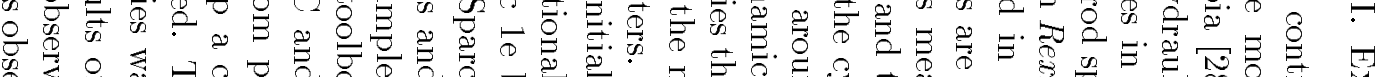

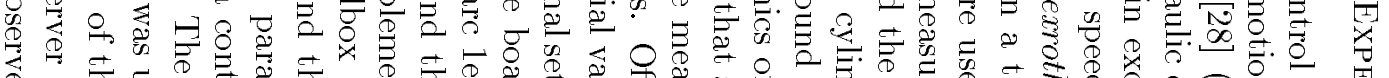

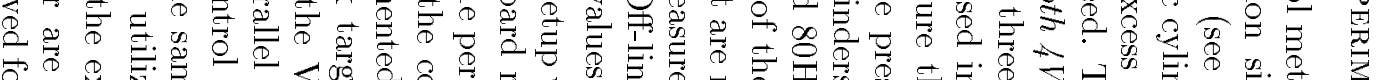

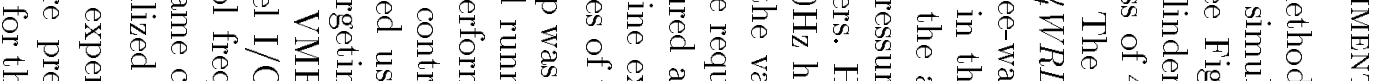

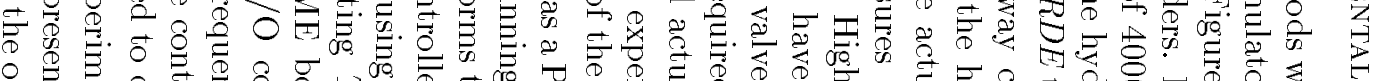

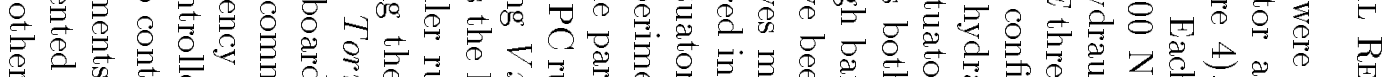

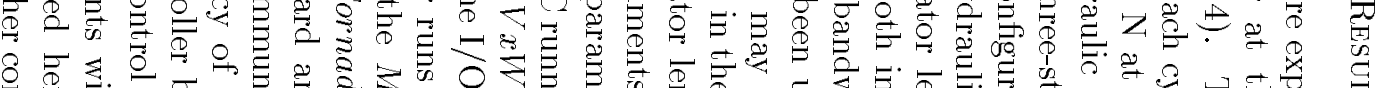

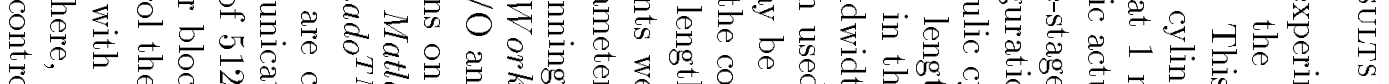

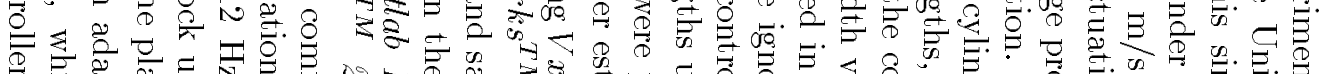
-

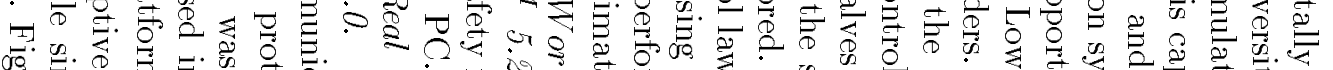

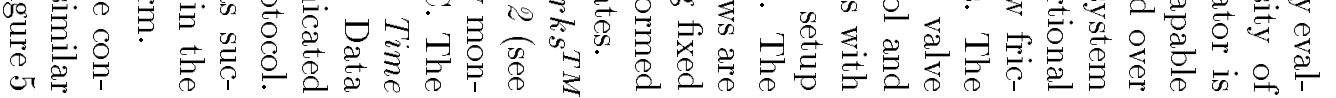

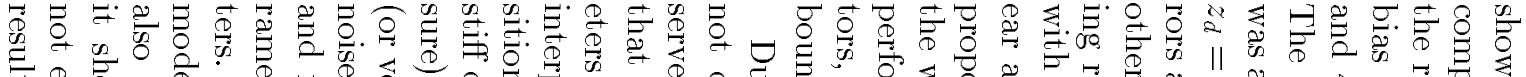

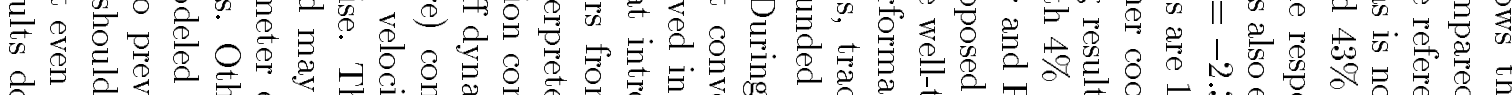

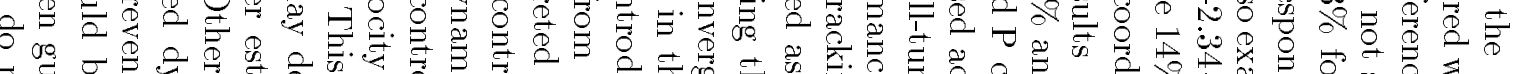

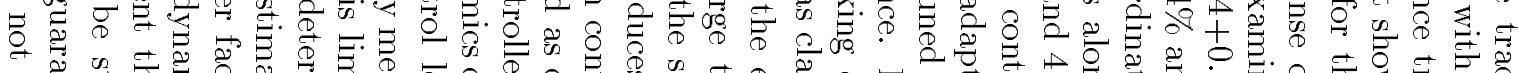

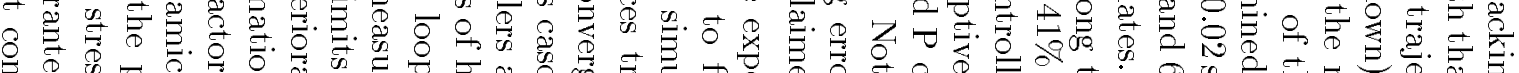

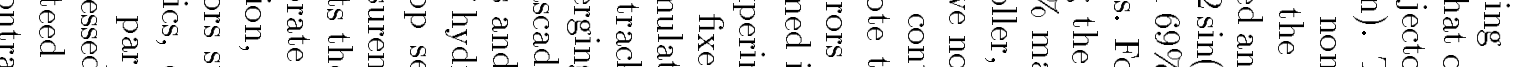

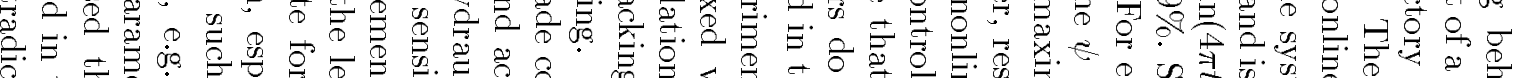

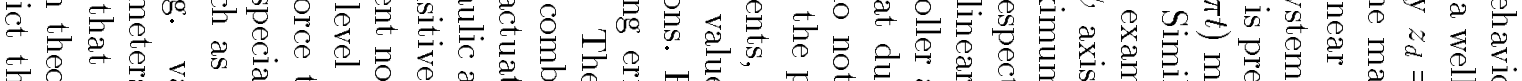

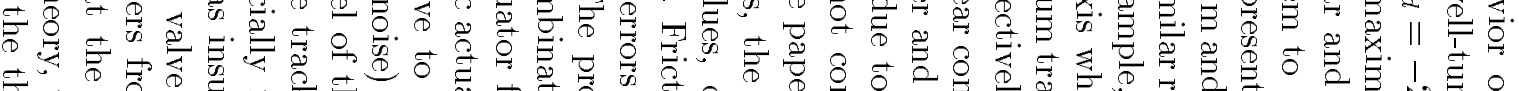

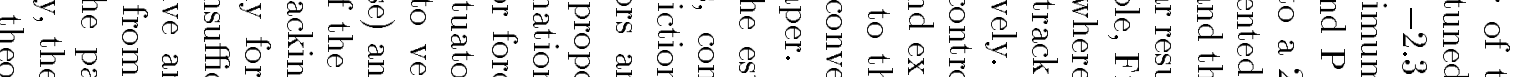

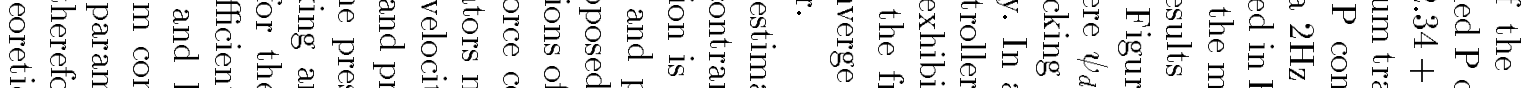

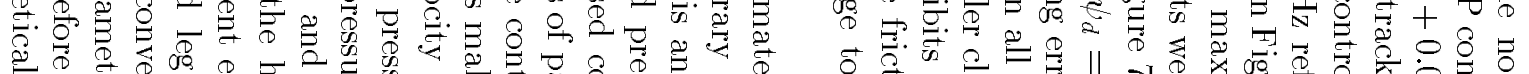

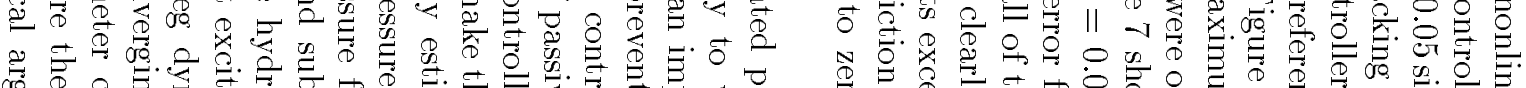

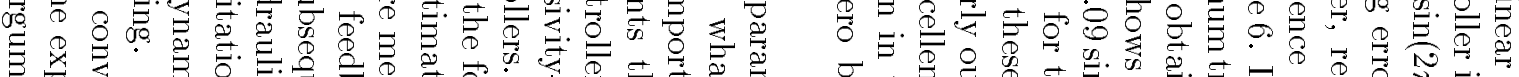

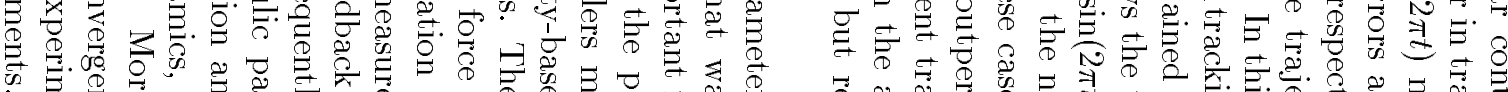

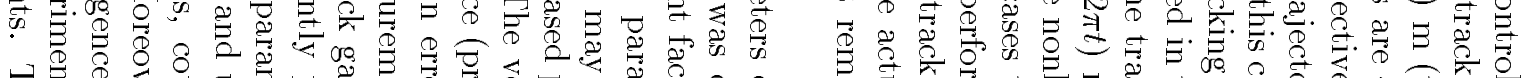

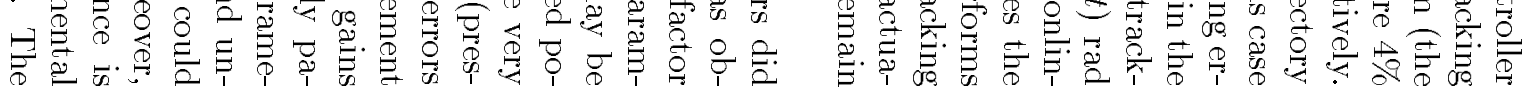
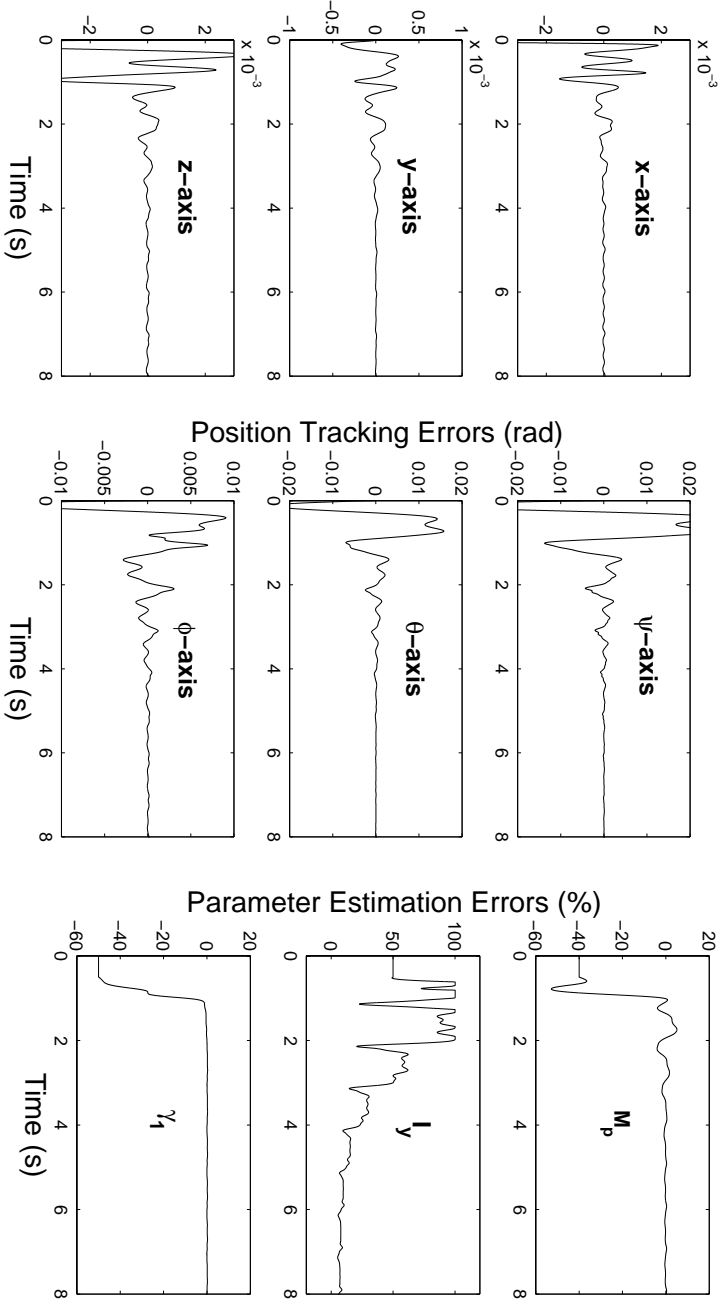

Parameter Estimation Errors (\%)

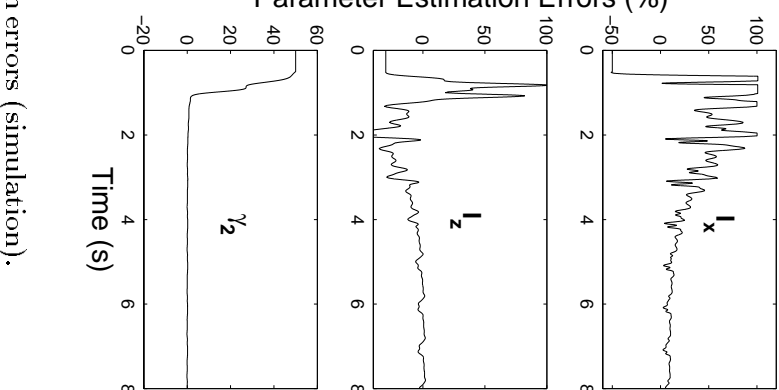




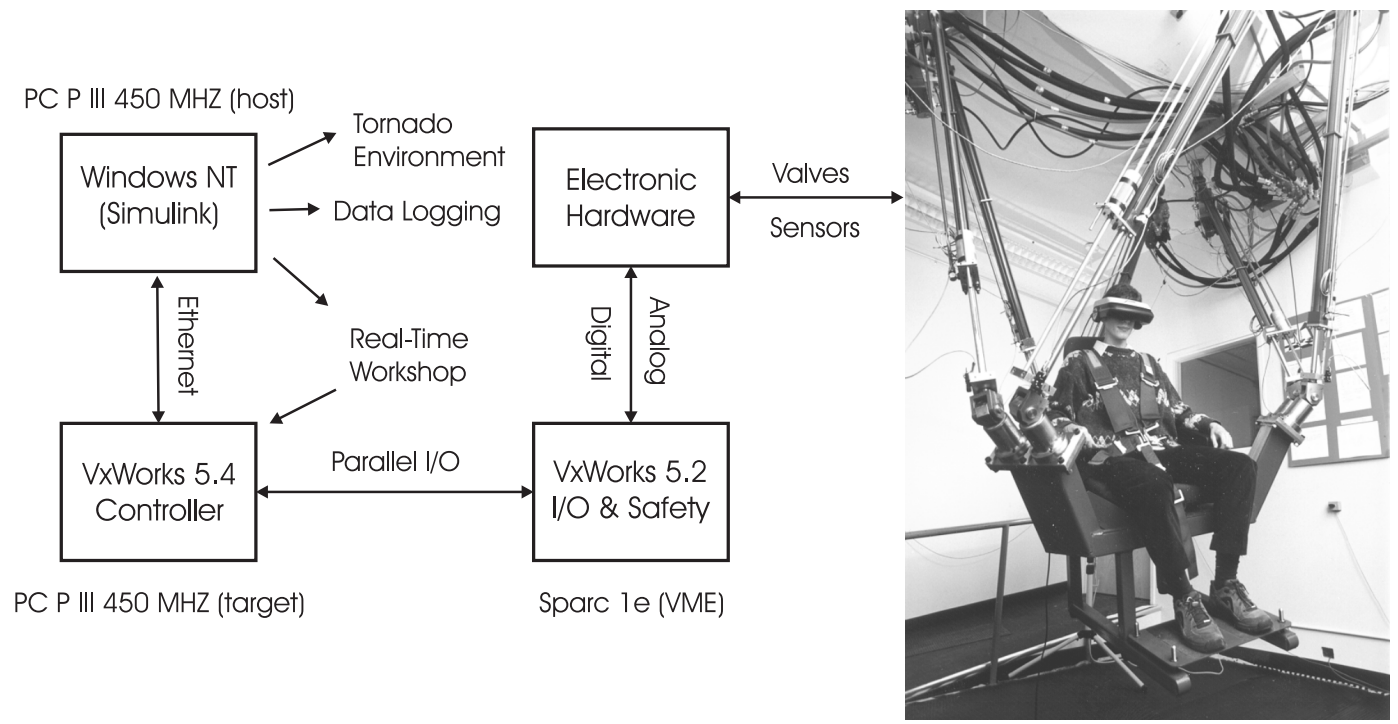

UBC Stewart Platform

Fig. 4. The experimental setup.

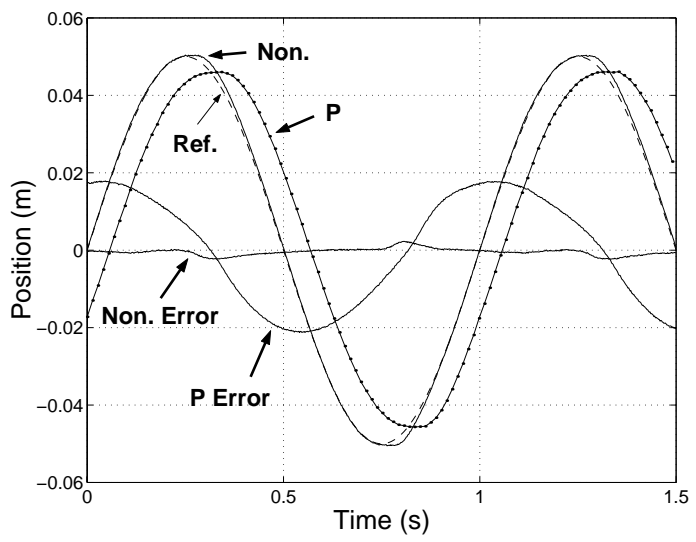

Fig. 5. Position tracking $(1 \mathrm{~Hz})$ along $z$ coordinate (experiment).

adaptation was found to be quite helpful in improving the tracking performance. The projection gains used in the adaptation laws proved effective in preventing the large parameter swings that can occur especially during startup transients. The step response of the controller along the $z$ axis is also compared with that of the $\mathrm{P}$ controller in Figure 8. As it can be seen $\Gamma$ the nonlinear controller exhibits a much faster response with some overshoot.

\section{CONCLUSIONS}

This paper addresses the control problem for hydraulically driven manipulators. The highly nonlinear dominant actuator dynamics prevents the use of standard robot control methods. In fact Tinclusion of actuator dynamics in the design is of critical importance in hydraulic robots. While most of the reported work in the literature consider the control of single-rod hydraulic actuators $\Gamma$ this paper proposed novel nonlinear controllers for hydraulic manipulators using backstepping. A realistic model of the system was uti-

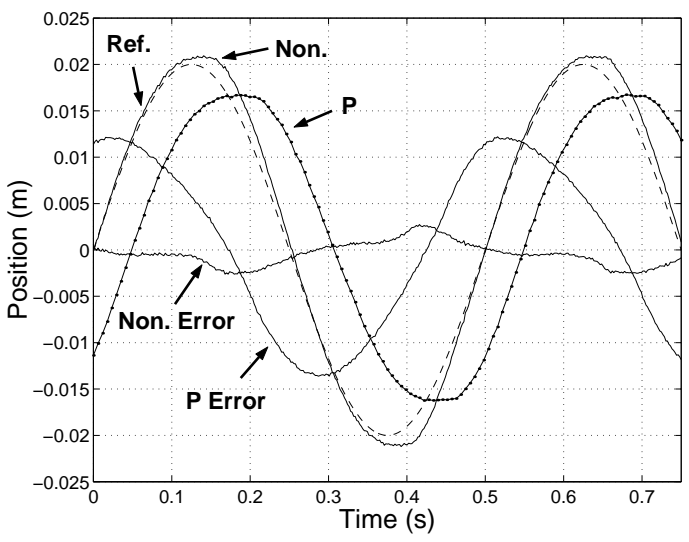

Fig. 6. Position tracking (2Hz) along $z$ coordinate (experiment).

lized in developing these Lyapunov-based controllers. To deal with parameter uncertainties Tthe controllers were augmented with adaptation laws. Acceleration feedback was avoided by proposing adaptive and sliding-type observers. Simulations and experiments were carried out with a hydraulic Stewart platform to investigate the effectiveness of these approaches. The results demonstrated excellent tracking position tracking behavior and satisfactory transient responses for these new controllers.

\section{Acknowledgments}

The authors would like to thank Wen-Hong Zhu for his technical comments $\Gamma$ Ruediger Six for his contribution to the real-time implementation of the controllersTand Simon Bachmann for his assistance in maintenance and modification of the hydraulic system. This work was supported by the Canadian IRIS/PRECARN Network of Centers of Excellence. 


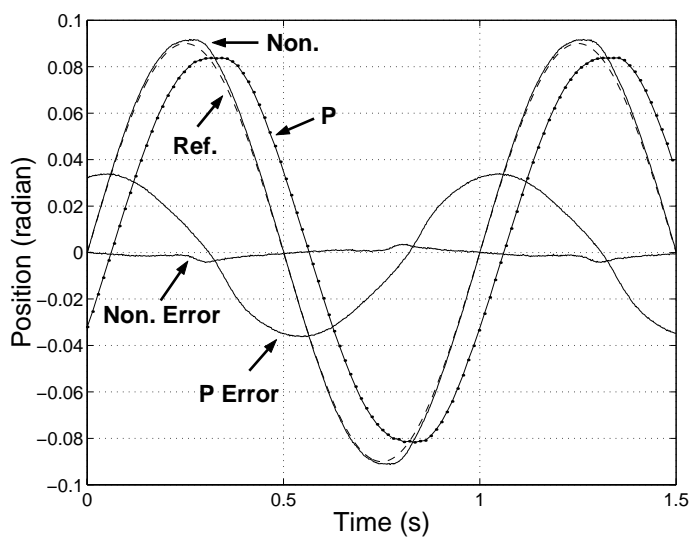

Fig. 7. Position tracking $(1 \mathrm{~Hz})$ along $\psi$ coordinate (experiment).

\section{Appendix A}

The dynamics of a typical hydraulic actuator are presented in this Appendix. A three-way valve configuration is assumed to be used in the actuatorsTas shown in Figure 9. For such a configurationTthe control pressure dynamics are governed by [29]

$$
\frac{V_{t}}{\beta} \dot{p}_{c}=q_{l}+c_{l}\left(p_{s}-p_{c}\right)-\dot{V}_{t}
$$

where $V_{t}$ is the trapped fluid volume in the control side $\Gamma$ $\beta$ is the effective bulk modulus $\Gamma p_{c}$ is the control pressure acting on the control side $\Gamma p_{s}$ is the supply pressure acting on the rod side $\Gamma q_{l}$ is the load flow $\Gamma$ and $c_{l}$ is the coefficient of total leakage. The load flow $\Gamma q_{l} \Gamma$ is a nonlinear function of the control pressure and the valve spool position and is given by

$$
q_{l}= \begin{cases}c(u-d) \sqrt{p_{c}} & u<-d \\ c(u+d) \sqrt{p_{s}-p_{c}}+c(u-d) \sqrt{p_{c}} & -d \leq u \leq d \\ c(u+d) \sqrt{p_{s}-p_{c}} & u>d\end{cases}
$$

and $c=c_{d} w \sqrt{\frac{2}{\rho}} \Gamma$ where $c_{d}$ is the effective discharge coefficient $\Gamma w$ is the port width of the valve $\rho$ is the density of the fluid $d$ is the valve underlap length and $u$ is the valve spool position which is the control command. Note that the actuator output force is $\tau=p_{c} A-p_{s} a$. Therefore $\Gamma$ using (56) and (57) Tthe dynamics of the $i$ 'th hydraulic actuator can be written in the following form (assuming $c_{l} \approx 0$ )

$$
\dot{\tau}^{i}=-\frac{A \beta^{i} \dot{q}^{i}}{q^{i}-l^{i}}+\frac{\beta^{i}}{q^{i}-l^{i}} q_{l}^{i}\left(\tau^{i}, u^{i}\right)=f^{i}\left(q^{i}, \dot{q}^{i}\right)+g^{i}\left(q^{i}, \tau^{i}, u^{i}\right)
$$

where $l$ is the actuator stroke length. For a Stewart platform $T$ there are six actuators driving the system. The actuator subsystem dynamics can be represented in matrix form as in (2).

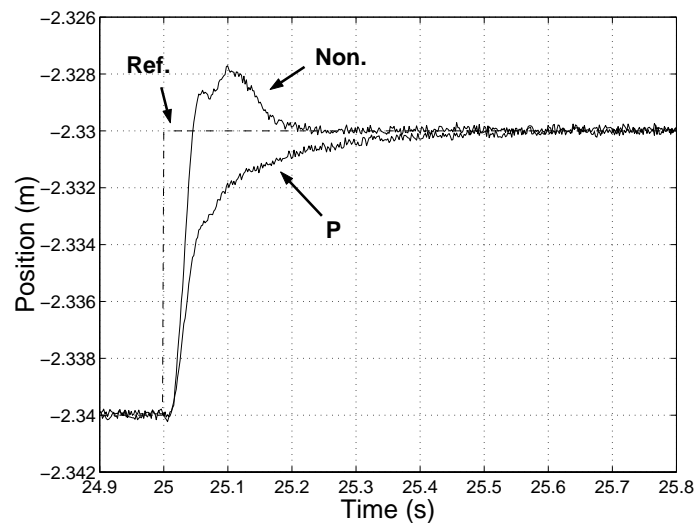

Fig. 8. Step response along $z$ coordinate (experiment).

Note that (58) can be rewritten in the following form which is suitable for adaptive control:

$$
\dot{\tau}^{i}=\gamma_{1}^{i} f_{0}^{i}\left(q^{i}, \dot{q}^{i}\right)+\gamma_{2}^{i} g_{0}^{i}\left(q^{i}, \tau^{i}, u^{i}\right)
$$

where $\gamma^{i}=\left[\begin{array}{ll}\beta^{i} & \beta^{i} c^{i}\end{array}\right]^{T} \Gamma f_{0}^{i}=-\frac{A^{i} \dot{q}^{i}}{q^{2}-l^{2}} \Gamma$ and $g_{0}^{i}=\frac{q_{l}^{i}}{c_{i}\left(q^{i}-l^{2}\right)}$ (does not depend on $c_{i}^{s}$ Tsee $(57)$ ). These equations can be written in matrix form as in (16).

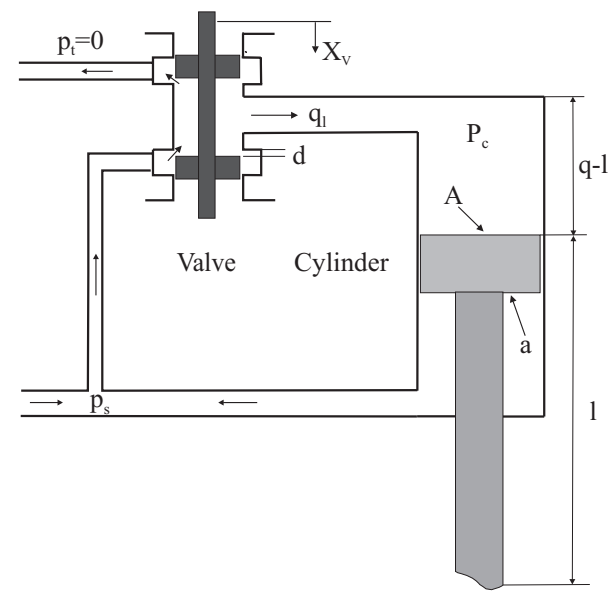

Fig. 9. A typical three-way valve configuration.

\section{Appendix B}

The Stewart platform is a parallel manipulator widely used in conventional motion simulators. The dynamics of an inverted ceiling-mounted Stewart platform (Figure (10)) are presented here [19].

In task-space coordinates $T$ the dynamics of the platform are governed by:

$$
D(q) \ddot{q}+C(q, \dot{q}) \dot{q}+G=(J L)^{T} \tau
$$

where $q=\left[\begin{array}{llllll}x & y & z & \psi & \theta & \phi\end{array}\right]^{T}$ and $\phi \Gamma \theta$ and $\psi$ are rollpitch-yaw anglesTrespectively. Furthermore $J$ is the manipulator Jacobian matrix and $L$ is defined as

$$
L=\left[\begin{array}{cc}
I_{3 \times 3} & 0 \\
0 & T
\end{array}\right]
$$


TABLE I

THE SYSTEM PARAMETERS USED IN THE SIMULATIONS AND EXPERIMENTS.

\begin{tabular}{|c|c|c|c|c|c|c|c|}
\hline \multicolumn{7}{|c|}{ Hydraulic Parameters } \\
\hline Parameter & $A\left(\mathrm{~m}^{2}\right)$ & $a\left(\mathrm{~m}^{2}\right)$ & $L(\mathrm{~m})$ & $P_{s}(\mathrm{psi})$ & $d(\mathrm{~m})$ & $c$ & $\beta(\mathrm{Mpa})$ \\
\hline Value & $1.14 \times 10^{-3}$ & $6.33 \times 10^{-4}$ & $1.37 \mathrm{~m}$ & 1500 & $55.4 \times 10^{-6}$ & $1.5 \times 10^{-4}$ & 700 \\
\hline \multicolumn{7}{|c|}{ Rigid Body Parameters } \\
\hline Parameter & $M_{p}(\mathrm{~kg})$ & $I_{x}\left(\mathrm{~kg} \cdot \mathrm{m}^{2}\right)$ & $I_{y}\left(\mathrm{~kg} \cdot \mathrm{m}^{2}\right)$ & $I_{z}\left(\mathrm{~kg} . \mathrm{m}^{2}\right)$ & & - \\
\hline Value & 250 & 45 & 45 & 43 & & - \\
\hline
\end{tabular}

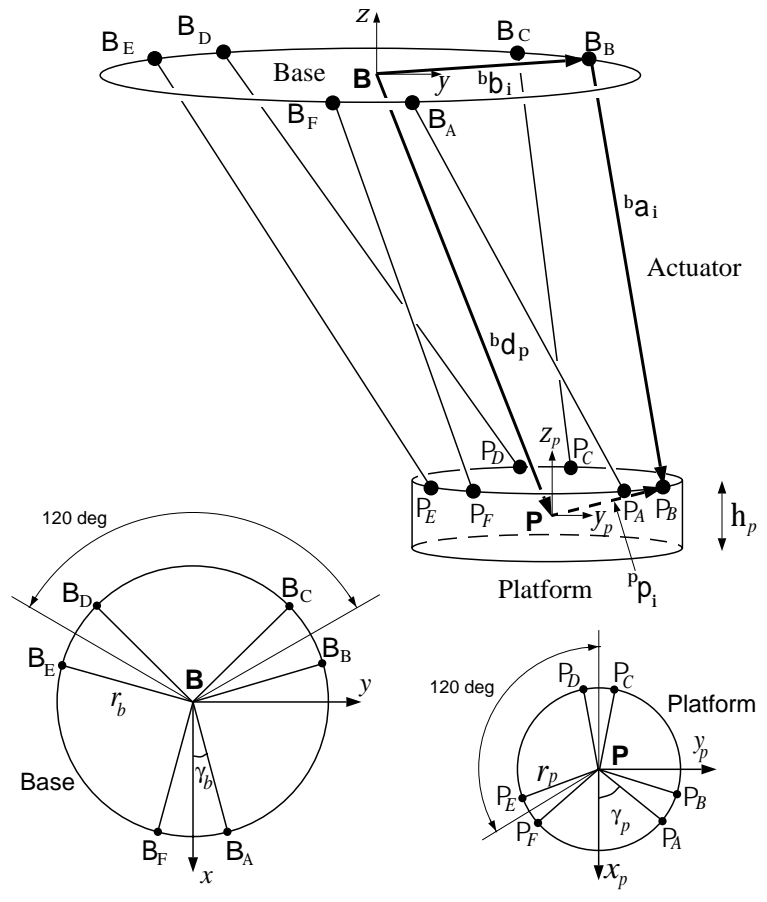

Fig. 10. The schematic of the Stewart platform.

with

$$
T=\left[\begin{array}{ccc}
\cos (\theta) \cos (\phi) & -\sin (\phi) & 0 \\
\cos (\theta) \sin (\phi) & \cos (\phi) & 0 \\
-\sin (\theta) & 0 & 1
\end{array}\right]
$$

Finally $D(q) \Gamma C(q, \dot{q})$ and $G$ have the following forms:

$$
\begin{aligned}
D(q) & =\left[\begin{array}{cc}
M_{p} I_{3 \times 3} & 0 \\
0 & T^{T b} I_{p} T
\end{array}\right] \\
C(q, \dot{q}) & =\left[\begin{array}{cc}
0 & 0 \\
0 & c_{22}
\end{array}\right] \\
c_{22} & =T^{T} S(\omega)^{b} I_{p} T+T^{T b} I_{p} \dot{T} \\
G & =\left[\begin{array}{llllll}
0 & 0 & M_{p} g & 0 & 0 & 0
\end{array}\right]^{T}
\end{aligned}
$$

where $\omega$ is the angular velocity vector of the platform and

$$
S(\omega)=\left[\begin{array}{ccc}
0 & -\omega_{z} & \omega_{y} \\
\omega_{z} & 0 & -\omega_{x} \\
-\omega_{y} & \omega_{x} & 0
\end{array}\right]
$$

In the above equations $\Gamma^{b} I_{p}$ is the platform inertia matrix with respect to the base frame and is given by

$$
{ }^{b} I_{p}=R{ }^{p} I_{p} R^{T}
$$

where

$$
{ }^{p} I_{p}=\left[\begin{array}{ccc}
I_{x} & 0 & 0 \\
0 & I_{y} & 0 \\
0 & 0 & I_{z}
\end{array}\right]
$$

and $R$ is a rotation matrix giving the coordinates of the platform-attached basis vectors in a base frame. Note that (60) is not exactly as (1). However $\Gamma$ since $J$ is a function of platform position and is knownT the controllers can be easily modified to be used in this case. Moreover Tthe rigid body dynamics may be written in so-called linear in parameters form.

$$
D(q) \ddot{q}+C(q, \dot{q}) \dot{q}+G=Y_{6 \times 4}(q, \dot{q}, \ddot{q}) \theta
$$

where $\theta=\left[\begin{array}{llll}M_{p} & I_{x} & I_{y} & I_{z}\end{array}\right]^{T}$ is the vector of unknown parameters. The detailed expressions of the elements of $Y$ are long and fairly straightforward and will not be presented here.

\section{REFERENCES}

[1] J.Y.S. Luh, M.W. Walker, and R.P.C. Paul, "Resolved acceleration control of mechanical manipulators," IEEE Tran. Automat. Cont., vol. 25, pp. 468-474, 1980.

[2] R. Ortega and M.W. Spong, "Adaptive motion control of rigid robots: A tutorial," Automatica, vol. 25, pp. 877-888, 1989.

[3] H. Berhuis and H. Nijmeijer, "A passivity approach to controllerobserver design for robots," IEEE Tran. Robot. Automat, vol. 9, no. 6, pp. 740-754, 1993.

[4] B. Paden and R. Panja, "Globally asymptotically stable 'PD+' controller for robot manipulators," Int. J. Control, vol. 47, pp. $1697-1712,1988$.

[5] J. J.-E Slotine and W. Li, "On the adaptive control of robot manipulators," Int. J. Robot. Research, vol. 6, pp. 49-59, 1987.

[6] M. W. Spong, "On the robust control of robot manipulators," IEEE Tran. Automat. Cont., vol. 37, pp. 1782-1786, 1992.

[7] S. Zenieh and M. Corless, "Simple robust tracking controllers for robotic manipulators," in SYROCO'94, September 1994, pp. 193-198.

[8] C. Abdallah, D. Dawson, P. Dorato, and M. Jamshidi, "Survey of robust control for rigid robots," IEEE Control Systems, pp. $24-30,1991$.

[9] T. J. Tarn, A. K. Bejczy, X. Yun, and Z. Li, "Effect of motor dynamics on nonlinear feedback robot arm control," IEEE Tran. Robot. Automat, vol. 7, no. 1, pp. 114-122, February 1991.

[10] J. Yuan, "Adaptive control of robotic manipulators including motor dynamics," IEEE Tran. Robot. Automat., vol. 11, no. 4, pp. 612-617, 1995.

[11] T. Burg, D. Dawson, J. Hu, , and M. de Queiroz, "An adaptive partial state-feedback controller for RLED robot manipulators," 
IEEE Tran. Automat. Contr., vol. 41, no. 7, pp. 1024-1030, July 1996.

[12] C. Y. Su and Y. Stepanenko, "Redesign of hybrid adaptive/robust motion control of rigid-link electrically-driven robot manipulator," IEEE Tran. Robot. Automat, vol. 14, no. 4, pp. 651-655, 1998.

[13] M. R. Sirouspour and S. E. Salcudean, "On the nonlinear control of hydraulic servo-systems," in Int. Conf. Robot. Automat., April 2000, pp. 1276-1282.

[14] G. A. Sohl and J. E. Boborow, "Experiments and simulations on the nonlinear control of a hydraulic servosystem," IEEE Tran. Cont. Syst. Tech., vol. 7, no. 2, pp. 238-247, March 1999.

[15] G. Vossoughi and M. Donath, "Dynamic feedback linearization for electrohydraulically actuated control systems," J. Dyn. Sys., Meas. and Contr., vol. 117, pp. 468-477, Dec. 1995.

[16] B. Novel, M.A. Garnero, and A. Abichou, "Nonlinear control of a hydraulic robot using singular perturbations," in Int. Conf. Syst. Man and Cyber., 1994, pp. 1932-1937.

[17] K. A. Edge and F. Gomes de Almeida, "Decentralized adaptive control of a directly driven hydraulic manipulator Part I: theory," in Instn. Mech. Engr., 1995, vol. 209, pp. 191-196.

[18] K. A Edge and F. Gomes de Almeida, "Decentralized adaptive control of a directly driven hydraulic manipulator Part II: experiments," in Instn. Mech. Engr., 1995, vol. 209, pp. 197-202.

[19] D. Li and S. E. Salcudean, "Modeling, simulation and control of a hydraulic Stewart platform," in IEEE Int. Conf. Robot. Automat., April 1997, pp. 3360-3366.

[20] F. Bu and B. Yao, "Observer based coordinated adaptive robust control of robot manipulators driven by single-rod hydraulic actuators," in IEEE Int. Conf. Robot. Automat., April 2000, pp. 3034-3039.

[21] M. Krstic, I. Kanellakopoulos, and P. Kokotovic, Eds., Nonlinear and Adaptive Control Design, Wiley and Sons, New York, 1995.

[22] Z. Pan and T. Basar, "Adaptive controller design for tracking and disturbance attenuation in parameteric strict-feedback nonlinear systems," IEEE Tran. Automat. Control, vol. 43, no. 8, pp. 1066-1083, August 1998.

[23] H. J. Shieh and K K Shyu, "Nonlinear sliding-mode torque control with adaptive backstepping approach for induction motor drive," IEEE Tran. Indus. Elect., vol. 46, no. 2, pp. 380-389, April 1999.

[24] W.E. Dixon, E. Zergeroglu, D.M. Dawson, and M.W. Hannan, "Global adaptive partial state feedback tracking control of rigidlink flexible-joint robots.," Robotica, vol. 18, pp. 325-336, MayJune 2000 .

[25] C. C. de Wit and J. J. E. Slotine, "Sliding observers for robot manipulators," Automatica, vol. 27, no. 5, pp. 859-864, 1991.

[26] J. E. Slotine and W. Li, Eds., Applied Nonlinear Control, Prentice-Hall Inc., New Jersey, 1991.

[27] W. H. Zhu and J. De Schutter, "Adaptive control of mixed rigid/flexible joint robot manipulators based on virtual decomposition," IEEE Tran. Robot. Automat., vol. 15, no. 2, pp. 310317, April 1999.

[28] S. E. Salcudean, P.A. Drexel, D. Ben-Dov, A.J. Taylor, and P.D. Lawrence, "A six degree-of-freedom, hydraulic, one person motion simulator," in Int. Conf. Robot. Automat, May 1994, pp. $859-864$.

[29] H. E. Merrit, Ed., Hydraulic control systems, Wiley and Sons, New York, 1967

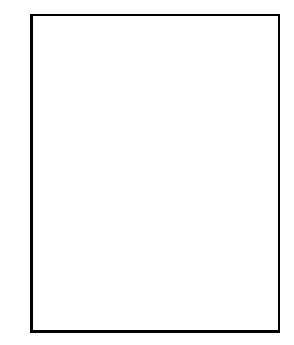

Septimiu E. Salcudean received the B. Eng and M.Eng degress from McGill University and the Ph.D. degree from U.C. Berkeley, all in Electrical Engineering. From 1986 to 1989 , he was a Research Staff Member in the robotics group at the IBM T.J. Watson Research Center. He then joined the Department of Electrical and Computer Engineering at the University of British Columbia, Vancouver, Canada, where he is now a Professor and holds a Canada Research Chair. He spent one year at ONERA in Toulouse, France, in 1996-1997, where he held a Killam Research Fellowship. Dr. Salcudean is interested in haptic interfaces, teleoperation and virtual environments. He is pursuing applications to medical diagnosis and intervention and to the control of heavy-duty hydraulic machines such as excavators. 